\title{
Outcomes after A1 Pulley Resection versus Release for Trigger Finger
}

\author{
Porter Young, Mohamed Salar*, Price Sessums, Jeb Williams, and Marc B. Kaye \\ Department of Orthopaedics and Rehabilitation, University of Florida-Jacksonville, USA
}

*Corresponding author: Mohamed Salar, Department of Orthopaedics and Rehabilitation, University of Florida-Jacksonville, USA.

Received Date: March 18, 2020

Published Date: April 06, 2020

\begin{abstract}
A1 pulley excision is a novel surgical technique that involves removing the entire pulley as opposed to merely dividing it. This may provide a solution for patients who are at higher risk of scarring or redevelopment of the pulley tissue, such as patients with diabetes. This study aims to compare outcomes of A1 pulley resection versus the standard release (or division) for patients with trigger finger. We hypothesize that complete resection of the A1 pulley will result in fewer instances of scarring and potential redevelopment of the pulley and subsequent postoperative referrals to occupational therapy (OT), steroid injections, or revision releases when compared to A1 pulley division only. A retrospective review was performed and 90 patients with 137 trigger finger releases were included in the study. Postoperative recovery comparisons as well as operative time were compared between the excision and division groups. Subgroup analysis was performed regarding outcomes of patients with diabetes and hypothyroidism. The excision group demonstrated improved outcomes compared to the incision group with lower rates of digital stiffness requiring OT referral ( $56 \%$ versus $61 \% \mathrm{p}=0.529)$, a lower rate of repeat injections ( $10 \%$ versus $20 \% \mathrm{p}=0.098$ ) and a lower rate of revision release $(2 \%$ versus $16 \% \mathrm{p}=0.004$ ). Average operative time was 4 minutes 10 seconds min longer in the excision group. Average follow up was 20.8 weeks in the excision group and 46.3 weeks in the incision group. Excision of the A1 pulley is a novel technique that may successfully reduce the rates of OT referrals and digital stiffness, but successfully reduces the need for postoperative injection, and most importantly, it reduces the incidence of revision surgeries with only a minor increase in operative time. We recommend this option for patients who are at an increased risk for redeveloping trigger finger.
\end{abstract}

Keywords: Trigger finger; Excision; A1 Pulley; Incision; Recurrence; Diabetes; Hypothyroidism

\section{Introduction}

Stenosing tenosynovitis is the entrapment of the hand's flexor tendons within their natural retinacular support, or pulley. It is commonly referred to as simply a trigger finger [1]. Trigger finger occurs when flexor tendons become mechanically impinged within these pulleys leading to painful catching, locking, and a potential eventual tendon rupture due to chronic wear [2-4]. This impingement can be explained by understanding the state of the inflamed tendon. The tenosynovitis causes swelling of the tendon sheath, tendon and pulley thickening and occasionally formation of a flexor tendon nodule ultimately leading to impingement with resultant painful clicking and locking [4-6]. This pathology can occur in isolation but can also occur in the setting of systemic disease. Diabetes and hypothyroidism are recognized as risk factors that put the patient at double the risk of developing stenosing tenosynovitis compared to the general population [7-9]. Despite its prevalence, it remains a common source of pain and disability. The scope of this pathology reaches its highest peak between the $5^{\text {th }}$ and $6^{\text {th }}$ decade of age[10]. There is a female to male predominance which in some studies reported to be as six times higher $[10,11]$. Several options are reported when managing this common hand problem. The conservative approach usually starts with the generalized measures such as rest, activity modification, NSAIDs, splinting, therapy, and corticosteroid injection targeting the inflamed tendon [12]. Frequently, nonsurgical management leads to complete resolution of symptoms. However, persisting symptoms may necessitate surgery. The open technique is a widely used method that is performed as an outpatient procedure in the United States [12]. In general, an incision is created over the A1 pulley of the affected digit, with dissection onto and exposing the A1 pulley. The pulley is divided longitudinally to allow the inflamed tendon to glide freely without further impedance [13]. 
However, with simple division, the pulley ends are still in close proximity, leading to potential reformation and recurrent symptoms, especially those with diabetes mellitus (Figure 1). Nonetheless, some subsets of patients, especially those with diabetes mellitus, develop a recurrence of the triggering symptoms after the surgical release. The prevalence of recurrent symptoms reported in some studies to be $>3 \%$ which ultimately required revisions surgery [1417]. In addition, patients with recurrent symptoms are more likely to have an increased number of corticosteroid injections, a higher number of occupational therapy referrals, and return to the OR for revision surgery. Therefore, identifying which patient group is more likely to have a complicated post-operative course is crucial. Furthermore, increasing health care costs demand improvement in any surgical techniques to fit the patients who are predictably at risk. Therefore, we hypothesize that the entire resection of the A1 pulley will lead to decrease recurrence of trigger finger by lowering the chance of pulley reformation by scar formation. This novel surgical technique may potentially lead to a decreased number of digital stiffness, therapy, injections, or revision surgeries.

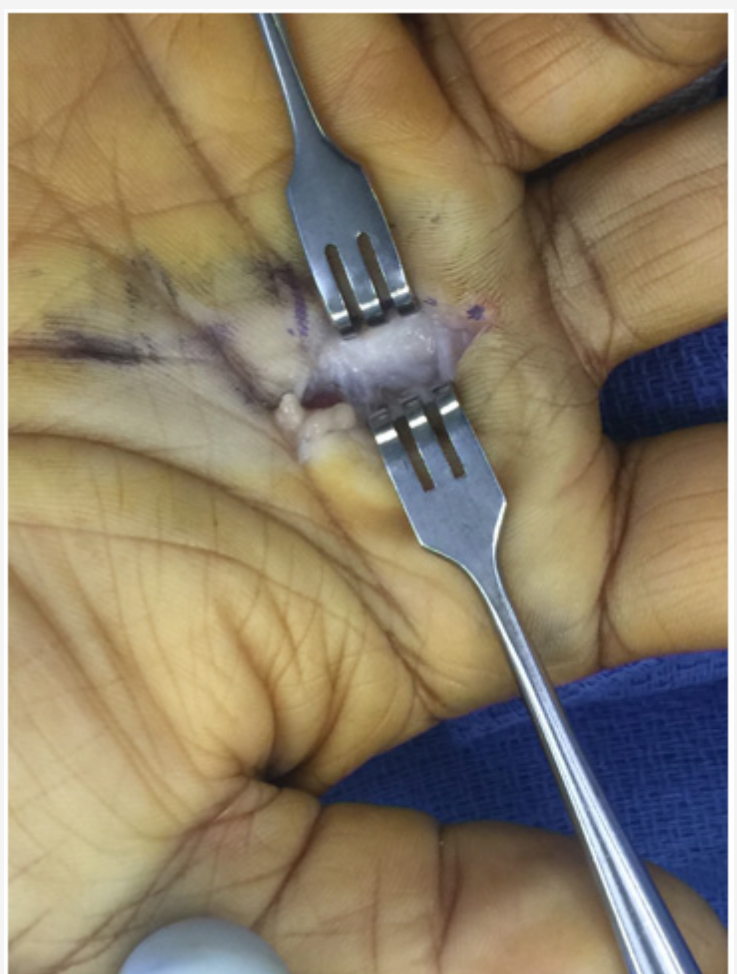

Figure 1: Right middle finger demonstrating a reformed and scarred A-1 pulley after a prior traditional A-1 pulley division.

\section{Materials and Methods}

A retrospective chart review was performed by identifying all patients who underwent trigger finger release by current procedural terminology (CPT) code 26055 (tendon sheath incision, e.g., for trigger finger) at our institution from $1 / 1 / 2015$ to $1 / 1 / 2018$. Operative reports were reviewed to determine which technique was utilized and operative time. Clinic notes were examined to determine the postoperative incidence of referrals to OT for digital stiffness, injections, and revision release in the previously treated digit. Patients were excluded if they underwent A1 pulley release for non-elective reasons such as infection as well as those lost to follow up. Ninety patients (totaling 137 digits) met our study criteria and were included in the study.

Univariate and multivariable analyses were performed to evaluate individual associations. Categorical variables were summarized using counts and percentages and analyzed using Chisquare or Fisher's tests. Some patients in the data set had multiple encounters during the data collection period; therefore, repeated measure models were fit to these data in the multivariable analyses. Generalized linear mixed data sets were used to determine whether factors such as excision and diabetes are associated with the outcomes. Five different variance-covariance structures (including first-order auto-regressive, compound symmetry, and "unstructured") were fit, and the structure with the smallest corrected Akaike Information Criterion (AICC) was selected. All statistical models were fit using SAS® 9.4.

\section{Proposed Surgical Technique}

The procedure is performed with the patient supine and the affected upper extremity on a hand table. A forearm tourniquet is utilized. The extremity is prepped and draped in a sterile fashion. A $2 \mathrm{~cm}$ longitudinal incision is marked out over the affected A-1 pulley, and local anesthetic is injected into the subcutaneous tissue. The skin is incised and blunt dissection utilizing tenotomy scissors is used to expose the A-1 pulley. Senn retractors are used to protect the digital neurovascular bundle running longitudinally along each side of the flexor tendon sheath. With the entire length of the pulley exposed and neurovascular structures protected, the longitudinal margins of the pulley are sharply excised with a fresh 15 blade scalpel (Figure 2). If the pre-pulley sheath is thickened as well, it is also included in the excision.

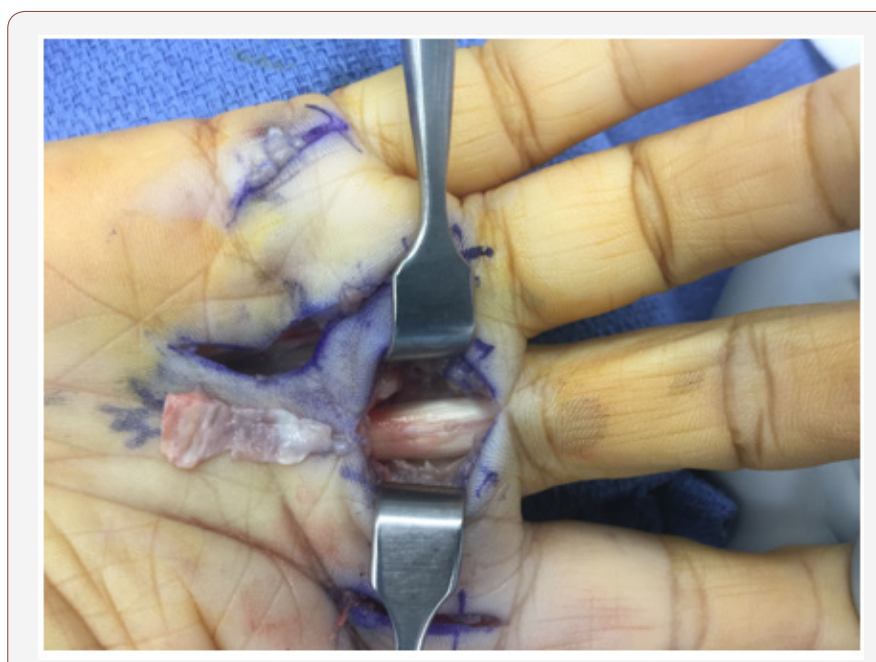

Figure 2: Right middle finger after A-1 pulley excision demonstrating resected pulley. The index and small fingers also underwent pulley excision. The ring finger demonstrates a revision A-1 pulley release which required an extended incision for release of adhesions from prior division of the pulley. 
Once the pulley is excised, the flexor tendons are mobilized with a ragnell retractor to ensure the tendon is gliding freely. The tourniquet is then released, bleeding is controlled with bipolar cautery and the skin is closed with suture in an interrupted fashion. A lightweight dry dressing is applied. Early, active range of motion is encouraged postoperatively to prevent stiffness and adhesion formation.

When performing this technique, it is important to ensure the neurovascular bundles are always protected as excising the peripheral edges of the pulley may place them at increased risk for

Table 1: Patients' Baseline Characteristics

\begin{tabular}{|c|c|c|c|c|c|}
\hline Variable & Category & $E(n=59,66 \%)$ & I $(n=31,34 \%)$ & Overall $(n=90)$ & P-Value \\
\hline \multirow{2}{*}{ Diabetes } & $\mathrm{N}$ & $30(51)$ & $18(58)$ & $48(53)$ & $0.514^{*}$ \\
\hline & $\mathrm{Y}$ & $29(49)$ & $13(42)$ & $42(47)$ & \\
\hline \multirow{2}{*}{ Gender } & $\mathrm{F}$ & $45(76)$ & $23(74)$ & $68(76)$ & $0.827^{*}$ \\
\hline & M & $14(24)$ & $8(26)$ & $22(24)$ & \\
\hline \multirow{2}{*}{ Thyroid Pathology } & $\mathrm{N}$ & $50(85)$ & $26(84)$ & $76(84)$ & $0.913^{* *}$ \\
\hline & $\mathrm{Y}$ & $9(15)$ & $5(16)$ & $14(16)$ & \\
\hline
\end{tabular}

${ }^{*}$ Pearson's Chi-square test; **Fisher's exact test

There was a significant difference

Table 2: Outcomes (All occurrences).

\begin{tabular}{|c|c|c|c|c|c|}
\hline Variable & Category & $\mathbf{E}(\mathrm{n}=\mathbf{8 8 , 6 4 \% )}$ & $\mathrm{I}(\mathrm{n}=\mathbf{4 9 , 3 6 \% )}$ & Overall $(\mathrm{n}=\mathbf{1 3 7})$ & P-Value \\
\hline \multirow{2}{*}{ Referred to OT } & $\mathrm{N}$ & $39(44)$ & $19(39)$ & $58(42)$ & $0.529^{*}$ \\
\cline { 2 - 6 } & $\mathrm{Y}$ & $49(56)$ & $30(61)$ & $79(58)$ & $118(86)$ \\
\hline \multirow{2}{*}{ Repeat Injection } & $\mathrm{N}$ & $79(90)$ & $39(80)$ & $19(14)$ & $0.098^{*}$ \\
\cline { 2 - 6 } & $\mathrm{Y}$ & $9(10)$ & $41(84)$ & $127(93)$ & $0.004^{* *}$ \\
\hline \multirow{2}{*}{ Revision Release } & $\mathrm{N}$ & $2(2)$ & $8(16)$ & $10(7)$ & \\
\cline { 2 - 6 } & $\mathrm{Y}$ & & & \\
\hline
\end{tabular}

*Pearson's Chi-square test; **Fisher's exact test

Table 3: Excision and diabetes with the outcomes.

\begin{tabular}{|c|c|c|c|}
\hline Outcome & Predictor & OR (95\%CI) & P \\
\hline \multirow{2}{*}{ Referred to OT } & Excision vs. Incision & $2.42(0.17,33.77)$ & 0.504 \\
\cline { 2 - 4 } & Diabetes yes vs. no & $0.74(0.05,11.11)$ & 0.82 \\
\hline Repeat Injection & Excision vs. Incision & $0.08(0.002,3.32)$ & 0.18 \\
\hline Revision Release & & & \\
\hline
\end{tabular}

Note: $\mathrm{OR}=$ Odds ratio; $\mathrm{Cl}=$ confidence interval

Table 4: Patients with diabetes only.

\begin{tabular}{|c|c|c|c|c|c|}
\hline Variable & Category & $E(n=45,68 \%)$ & I $(n=21,32 \%)$ & Overall $(n=66)$ & P-Value \\
\hline \multirow{2}{*}{ Referred_to_OT } & $\mathrm{N}$ & $21(47)$ & 7 (33) & $28(42)$ & $0.307^{*}$ \\
\hline & $\mathrm{Y}$ & $24(53)$ & $14(67)$ & $38(58)$ & \\
\hline \multirow{2}{*}{ Repeat_Injection } & $\mathrm{N}$ & 39 (87) & $14(67)$ & $53(80)$ & $0.094^{* *}$ \\
\hline & $\mathrm{Y}$ & $6(13)$ & $7(33)$ & $13(20)$ & \\
\hline \multirow{2}{*}{ Revision_Release } & $\mathrm{N}$ & $44(98)$ & $16(76)$ & $60(91)$ & $0.011^{* *}$ \\
\hline & $\mathrm{Y}$ & $1(2)$ & $5(24)$ & $6(9)$ & \\
\hline
\end{tabular}

*Pearson's Chi-square test; **Fisher's exact test 
Table $4 \& 5$ represent the endpoints association against diabetes by itself versus hypothyroidism alone. Despite the reduction in all measured outcomes, the excision group in the diabetic patients was statistically significant only in terms of reduction in required revision surgery.

Table 5: Patient with thyroid pathology.

\begin{tabular}{|c|c|c|c|c|c|}
\hline Variable & Category & $E(n=15,68 \%)$ & I $(n=7,32 \%)$ & Overall & P-Value \\
\hline \multirow{2}{*}{ Referred_to_OT } & $\mathrm{N}$ & $11(73)$ & $2(29)$ & $13(59)$ & \multirow{2}{*}{$0.074^{*}$} \\
\hline & $\mathrm{Y}$ & $4(27)$ & $5(71)$ & $9(41)$ & \\
\hline \multirow{2}{*}{ Repeat_Injection } & $\mathrm{N}$ & $13(87)$ & $7(100)$ & $20(91)$ & \multirow{2}{*}{$1.000^{*}$} \\
\hline & $\mathrm{Y}$ & $2(13)$ & $0(0)$ & $2(9)$ & \\
\hline Revision_Release & $\mathrm{N}$ & $15(100)$ & $7(100)$ & $22(100)$ & . \\
\hline All tests done using Fisher's & & & & & \\
\hline
\end{tabular}

*Fisher's exact test

\section{Discussion}

Trigger fingers remain one of the most common hand problems. It's typically a complaint for patients in their fifth and sixth decade of age $[10,11]$. Traditionally, management starts with a nonsurgical option such as NSAIDs, splinting, corticosteroid injections, and infrequently occupational therapy [12]. Once the conservative management fails, surgery is indicated [1-10]. This typically comprises division of the A1 pulley. Nonetheless, symptomatic recurrence or postoperative pain remain a concern to the treating surgeon. Prior studies noted a recurrence rate and revision surgery of trigger fingers around 3-12\% [14-17]. Keep in mind, that patients who return with locking and triggering of their digits will ultimately translate into increased office visits, the number of corticosteroid injection, and referral to OT [14-17]. If the second trial of conservative management fails, then a return to the operating room is likely. In an atmosphere where health care costs continue to rise, this issue persists as a burden on the health care system.

Previous publications demonstrated diabetes mellitus (DM) and hypothyroid disease as known risk factors for acquiring primary triggering [7-9]. Such patients are two times more likely to develop trigger fingers compared to the general population [7-9]. Furthermore, DM has always been a risk factor for various post-surgical complications. Trigger finger release surgery is not an exception [18]. These complications range from wound healing problems, infection, scar formation, and recurrence of symptoms [18]. Both DM and hypothyroidism are a common medical problem in the US adult population. Currently, there are 21 million American diagnosed with diabetes [19]. The prevalence of hypothyroid disease affects approximately one in every ten adult patients [20]. There are no studies addressing these medical problems role in recurrent digit triggering following surgical release. Therefore, we designed this study to compare a novel technique that deals with patients with DM and thyroid pathology. We proposed that patients with diabetes tend to reform their A1 pulley through fibrosis and scar formation. Therefore, excising rather than incising the A1 pulley will result in less likelihood of a pulley reformation. A1 pulley shown in Figure 1 is an example of an excised A1 pulley despite a complete incision a year before. The results of this study showed that half of our patients' population were diabetics 47\% (42/90), while the prevalence of thyroid disease was 16\% (14/90). Prior studies indicated female to male predominance for trigger fingers and our study where no different [10-11].

There were 76\% (68/90) females with trigger fingers presented to our clinics vs. only $24 \%$ (22/90). The primary endpoints of our study were to investigate whether excision of the A1 pulley would ultimately lower the triggering recurrence, which was measured by the number of corticosteroid injection, referral to OT and need for revision surgery. As it was shown in Table 2 there were fewer revision surgeries in the excision group vs the incision group $2 \%(2 / 88)$, vs $16 \%(8 / 49)$ which was statistically significant $(\mathrm{P}=0.004)$. OT referrals and corticosteroid injections were not significantly affected by this new technique but appeared to be less with the excision group.

In conclusion, the goal of this study was to demonstrate that resection of the A1 pulley is associated with decreased recurrence rate measured by OT referrals, corticosteroid injection and most importantly return to the operating room for revision surgery. Excision group in the diabetic patients had reduced recurrence symptoms as demonstrated by the endpoints, and this change was statistically significant. Therefore, we recommend this novel technique to be considered when dealing with patients with stenosing tenosynovitis who have DM or have a thyroid pathology.

\section{Acknowledgment}

Center of Health Equity and Quality Research, University of Florida-Jacksonville

\section{Conflict of Interest}

No conflict of interest.

\section{References}

1. Ryzewicz M, Wolf JM (2006) Trigger digits: principles, management, and complications. J Hand Surg Am 31(1): 135-146.

2. Carlson CS, Curtis RM (1984) Steroid injection for flexor tenosynovitis. J Hand Surg Am 9(2): 286-287. 
3. Rayan GM (1990) Distal stenosing tenosynovitis. J Hand Surg Am 15(6): 973-975.

4. Rhoades CE, Gelberman RH, Manjarris JF (1984) Stenosing tenosynovitis of the fingers and thumb. Results of a prospective trial of steroid injection and splinting. Clin Orthop Relat Res 190: 236-238.

5. Newport ML, Lane LB, Stuchin SA (1990) Treatment of trigger finger by steroid injection. J Hand Surg Am 15(5): 748-750.

6. Sampson SP, Badalamente MA, Hurst LC, Seidman J (1991) Pathobiology of the human A1 pulley in trigger finger. J Hand Surg Am 16(4): 714-721.

7. Stahl S, Kanter Y, Karnielli E (1997) Outcome of trigger finger treatment in diabetes. J Diabetes Complicat 11(5): 287-290.

8. Chammas M, Bousquet P, Renard E, Poirier JL, Jaffiol C, et al. (1995) Dupuytren's disease, carpal tunnel syndrome, trigger finger, and diabetes mellitus. J Hand Surg Am 20(1): 109-114.

9. Griggs SM, Weiss AP, Lane LB, Schwenker C, Akelman E, et al. (1995) Treatment of trigger finger in patients with diabetes mellitus. J Hand Surg Am 20(5): 787-789.

10. Fahey JJ, Bollinger JA (1954) Trigger-finger in adults and children. J Bone Joint Surg Am 36-A(6): 1200-1218.

11. Weilby A (1970) Trigger finger. Incidence in children and adults and the possibility of a predisposition in certain age groups. Acta Orthop Scand 41(4): 419-427.

12. Patel MR, Bassini L (1992) Trigger fingers and thumb: when to splint, inject, or operate. J Hand Surg Am 17(1): 110-113
13. Ryzewicz M, Wolf JM (2006) Trigger digits: principles, management, and complications. J Hand Surg Am 31(1): 135-146.

14. Fu YC, Huang PJ, Tien YC, Lu YM, Fu HH, et al. (2006) Revision of incompletely released trigger fingers by percutaneous release: results and complications. J Hand Surg Am 31(8): 1288-1291.

15. Huang HK, Wang JP, Lin CJ, Huang YC, Huang TF, et al. (2017) Short-term Versus Long-term Outcomes After Open or Percutaneous Release for Trigger Thumb. Orthopedics 40(1): e131-e135.

16. Benson LS, Ptaszek AJ (1997) Injection versus surgery in the treatment of trigger finger. J Hand Surg Am 22(1): 138-144.

17. Bruijnzeel H, Neuhaus V, Fostvedt S, Jupiter JB, Mudgal CS, et al. (2012) Adverse events of open A1 pulley release for idiopathic trigger finger. J Hand Surg 37(8): 1650-1656.

18. Adamina M, Kehlet H, Tomlinson GA, Senagore AJ, Delaney CP (2011) Enhanced recovery pathways optimize health outcomes and resource utilization: a meta-analysis of randomized controlled trials in colorectal surgery. Surgery 149(6): 830-840.

19. Bullard KM, Cowie CC, Lessem SE, Sharon H Saydah, Andy Menke, et al. (2018) Prevalence of diagnosed diabetes in adults by diabetes type United States, 2016. MMWR Morb Mortal Wkly Rep 67(12): 359-361.

20. Kostoglou Athanassiou I, Ntalles K (2010) Hypothyroidism - new aspects of an old disease. Hippokratia 14(2): 82-87. 